\title{
CORREDORES NATURALES, FUENTE DE OBSIDIANA Y ESTACIONALIDAD: EL CASO DE LA CIRCULACIÓN HUMANA ENTRE PALI AIKE Y SIERRA BAGUALES (PATAGONIA MERIDIONAL)
}

\author{
NATURAL CORRIDORS, SOURCE OF OBSIDIAN AND SEASONALITY: \\ THE CASE OF THE HUMAN MOVEMENT BETWEEN PALI AIKE AND \\ SIERRA BAGUALES (SOUTHERN PATAGONIA)
}

\author{
María Cecilia Pallo
}

\begin{abstract}
Los estudios líticos en el campo volcánico Pali Aike-CVPA, en el extremo sur de Patagonia Meridional Continental-han llevado a plantear una posible estrategia de aprovisionamiento directo de la obsidiana gris-verdosa veteada procedente de la sierra Baguales, a más de $200 \mathrm{~km}$ al norte del área. A partir del modelado de distintas variantes de superficies de fricción y el estrés invernal en un entorno SIG (Sistemas de Información Geográfica), se discute la existencia y disponibilidad anual de potenciales vías de circulación humana entre ambos espacios. Se sostiene que la interacción entre el CVPA y la sierra Baguales no puede ser explicada por una única vía de menor costo posible. En cambio, un pool de vías de bajo costo por sectores esteparios, cuya optimalidad fue variable en función de los cambios paleoambientales del Holoceno, parece ser la alternativa más defendible.
\end{abstract}

Palabras claves: circulación humana, fuente de obsidiana, SIG, extremo sur de Patagonia.

\begin{abstract}
Lithic studies in the Pali Aike volcanic field-PAVF, in the far south of Southern Continental Patagonia, have led to propose a possible direct provision strategy of banded greenish-gray obsidian from the Baguales range, more than $200 \mathrm{~km}$ north of the area. From the modeling of variants of friction surfaces and winter stress in a GIS environment (Geographic Information Systems), the existence and annual availability of potential routes of human movement between both spaces is discussed. It is argued that the interaction between the PAVF and the Baguales range could not be explained by a single route of lowest possible cost. Instead, a pool of low cost routes through steppe areas, the optimality of which depended on Holocene palaeoenvironmental changes, seems to be the more defensible alternative..
\end{abstract}

Key words: Human movement, obsidian source, GIS, southernmost continental Patagonia.

La obsidiana gris-verdosa veteada tiene su lugar de origen más probable en la sierra Baguales $\left(72^{\circ} \mathrm{W}\right.$ y $50,3^{\circ} \mathrm{S}$, Stern y Franco 2000$)$. Como parte de la zona limítrofe de altas cumbres entre Argentina y Chile, Baguales es una continuación de la cordillera de los Andes al sur del lago Argentino, con formas accidentadas y elevaciones que alcanzan los $1.800 \mathrm{msm}$. Solo en sitios próximos a este cordón montañoso la obsidiana registra frecuencias relativamente altas (Franco 2014; Stern y Franco 2000), mientras que en otros contextos -todos ellos al sur del río Santa Cruz- sus proporciones son muy escasas (Figura 1).

Al sur del cordón Baguales la obsidiana gris-verdosa veteada fue hallada en sitios de sus estribaciones sudoccidentales (Morano Büchner et al. 2009; San Román y Morello 1999, 2003).
Estos sitios presentan una tecnología similar a la registrada hacia el oriente (estancia La Verdadera Argentina -LVA-), aunque en estos últimos -a solo $30 \mathrm{~km}$ de Baguales y con avanzados estudios (p.ej., Borrazzo 2006, 2008; Borrero y Borrazzo 2011; Borrero et al. 2006)- la presencia de la obsidiana aún no ha sido confirmada ${ }^{1}$. Dentro de este contexto regional, para el área Laguna CóndorCVPA -en el extremo sur del continente- los análisis tecnológicos y la relativa abundancia de esta roca respecto de otras variedades de obsidiana llevaron a proponer la existencia de un posible sistema de aprovisionamiento directo desde el CVPA hacia la sierra Baguales (Charlin 2009; Charlin et al. 2011).

Dado este panorama regional nos interesa plantear expectativas sobre la circulación humana entre la sierra Baguales y el sector Laguna

1 CONICET-IMHICIHU (Instituto Multidisciplinario de Historia y Ciencias Humanas). Saavedra 15, 5º piso (1083 ACA), Buenos Aires, Argentina. ceciliapallo@gmail.com 


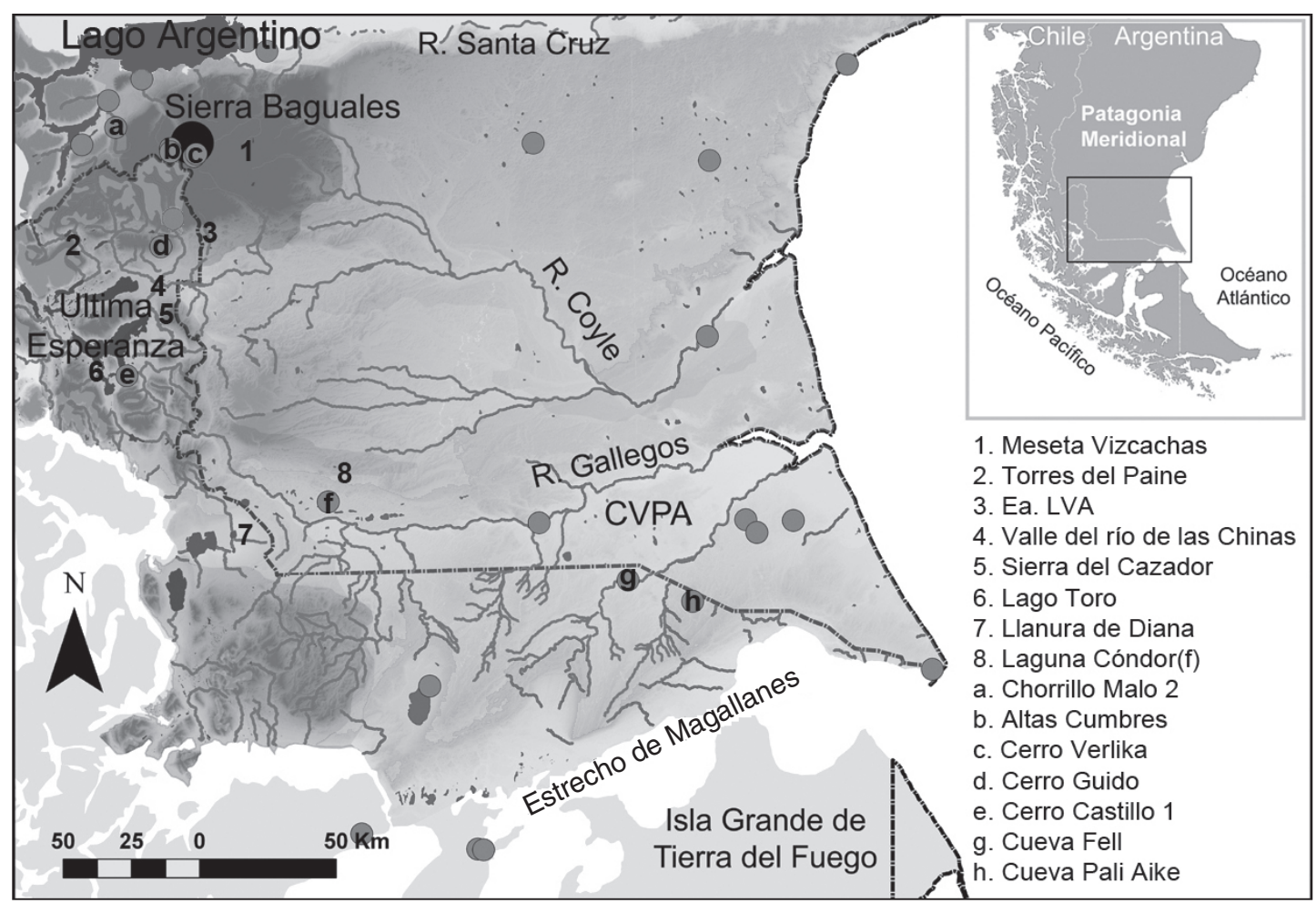

Figura 1. Área de estudio y distribución de sitios con artefactos en obsidiana gris-verdosa veteada (círculos grises). Study area and distribution sites with banded greenish-gray obsidian artifacts (grey circles).

Cóndor-CVPA, considerando aspectos vinculados con potenciales vías de paso pericordilleranas vs. esteparias y la disponibilidad estacional de los espacios involucrados. Los contrastes que puedan establecerse entre condiciones de transitabilidad, aislamiento, disponibilidad estacional y dificultad de acceso a la fuente en diferentes momentos del Holoceno serán centrales en esta discusión. Si bien no profundizamos en este tema, los modelos aquí generados avanzan en la discusión sobre diferentes circuitos de interacción con la sierra Baguales. Ambos circuitos estarían vinculados con dos esferas independientes de aprovechamiento directo de la fuente de obsidiana, una de ellas funcionando en las estribaciones occidentales del continente y la otra en las estepas orientales.

\section{Dinámica Ambiental y Circulación Humana}

Los criterios por los cuales los humanos realizan sus elecciones de uso y circulación por el espacio están vinculados en gran medida con la percepción de los costos y beneficios que encierra la dinámica ambiental (p.ej., Binford 1982; Kelly 1995). Desde esta perspectiva, el espacio adquiere sentido como un recurso en sí mismo, que incide de manera directa en la organización espacial humana (Borrero 1989-90). Esto hace esperable que las poblaciones tiendan a utilizar corredores que impliquen baja resistencia, favoreciendo el traslado con el menor gasto de energía posible (p.ej., Madry y Rakos 1996; Matteucci et al. 2011), aunque también podrían estar involucrados aspectos sociales, territoriales o simbólicos (Gamble 1998; Ingold 2000; Whallon 2006).

En la escala multi-anual del Holoceno, distintos elementos del paisaje debieron funcionar alternativamente como atractores o limitantes de la circulación humana hacia la sierra Baguales (Borrero et al. 2006; Borrero y Borrazzo 2011; Pafundi 2006). El macizo montañoso de Torres del Paine -al suroeste de Baguales, en Chile- presenta una morfología similar, pero con mayores alturas y volumen de cerros, lo que posiblemente haya implicado costos de circulación altos. Condiciones 
de acceso más favorables se reconocen hacia las estribaciones sudorientales, la meseta Vizcachas (Argentina) y los ambientes de estepa.

También la reconstrucción de las variaciones anuales de precipitación (Tonello et al. 2009), vegetación (Huber y Markgraf 2003; Mancini et al. 2011; Sottile et al. 2012; Villa-Martínez y Moreno 2007) y episodios de incendios en Patagonia Meridional (Huber y Markgraf 2003; Huber et al. 2004; Sottile et al. 2012) permiten estimar la existencia de paisajes vinculados con períodos más y menos favorables para la circulación humana hacia Baguales (Figura 2). La transición PleistocenoHoloceno está marcada por condiciones poco húmedas, gran ocurrencia de incendios y ambientes abiertos en sectores cordilleranos (Mancini et al. 2011; Tonello et al. 2009; Villa-Martínez y Moreno 2007). La facilidad de acceso hacia estos espacios pudo quedar restringida por las dimensiones del Gran Paleolago Tehuelche en Torres del Paine, que debió funcionar como un callejón sin salida desconectando sectores al norte y al sur de la sierra del Cazador.

De acuerdo con Solari y colaboradores (2012), avanzado el Holoceno Medio ocurrió el desagüe del paleolago por el seno de Última Esperanza -hace 7.113 cal a.p.-, aunque también podría haber ocurrido antes (García et al. 2014). Con el desarrollo del actual complejo hidrológico en Torres del Paine un nuevo aumento de la humedad permitió el establecimiento de bosques pericordilleranos más densos y la disminución de los espacios cubiertos por la estepa (Tonello et al. 2009; Villa-Martínez y Moreno 2007). La expansión gradual del bosque de Nothofagus también ha sido correlacionada con los avances glaciarios ocurridos a partir del 5.000 cal a.p. (Glasser et al. 2004; Moreno et al. 2009).
Es posible que estos factores junto con la altitud y la cercanía al Campo de Hielo Sur determinaran la existencia de sectores con condiciones climáticas y costos de circulación adversos para acceder a la sierra Baguales desde el sur. A partir de los 1.000 cal a.p. nuevas oportunidades para la circulación humana pudieron estar vinculadas con áreas cercanas a la cordillera, favorecidas por la declinación de las precipitaciones y la consecuente contracción del bosque (Tonello et al. 2009); aunque dicha tendencia se hizo más clara a fines del siglo XIX, con el asentamiento europeo (p.ej., Huber et al. 2004; Mancini et al. 2011; Villa-Martínez y Moreno 2007).

En una escala temporal más breve, episodios de sequía, deshielo, desborde de ríos, caída de nieve o congelamiento de suelos y ríos (Mayr et al. 2007; Paruelo et al.1998; Peláez y Jiménez 2006; Solari et al. 2012; Sturzenbaum y Borrelli 2001) debieron imponer procesos de obstrucción o fragmentación del hábitat, introduciendo cambios en los costos de uso y la oferta de los espacios transitables. Estas condiciones debieron ser el trasfondo de procesos humanos que involucraran estimaciones de costo/ beneficio, riesgo e incertidumbre, formas de acceso estacional, o bien reformulación de los circuitos de movilidad (p.ej., Avery 1995; Pafundi 2006; Pallo 2012; Veth 1993).

Si bien puede resultar difícil estimar el impacto real de la dinámica ambiental, las decisiones humanas usualmente involucran una relativa persistencia tanto en el uso de los espacios para transitar como en la imposibilidad de hacerlo (Beaton 1991). De esta manera, un acercamiento a las potenciales formas de la circulación entre los cazadores recolectores móviles de Patagonia Meridional adquiere importancia en el reconocimiento de la escala promediada del registro arqueológico durante el Holoceno.

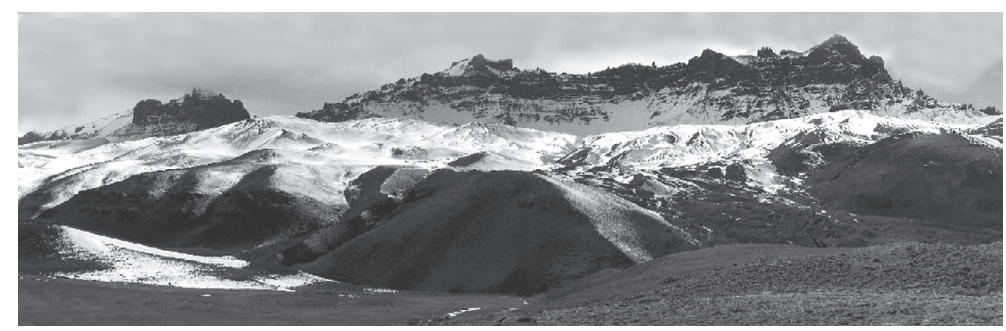

Figura 2. Estribaciones occidentales de la sierra Baguales (foto tomada y modificada de Ruiz y Doberti Limitada 2008).

Western foothills of the Baguales range (photograph extracted and modified from Ruiz and Doberti Limitada 2008). 


\section{Información Arqueológica y Etnohistórica}

La información temporal indica que la sierra Baguales debió ser explorada y conocida desde los inicios de la ocupación humana al sur del lago Argentino (Franco 2014). Las evidencias más tempranas de su explotación proceden del sitio Chorrillo Malo 2, cuya ocupación comienza en el 9.700 a.p. (Franco y Borrero 2003; Franco et al. 1999). De acuerdo con los estudios distribucionales, la sierra Baguales sería un área de uso marginal cuya principal actividad por parte de los humanos habría estado centrada en la obtención de la obsidiana gris-verdosa veteada, en el marco de ocupaciones que no eran prolongadas ni muy pautadas (Borrero y Carballo Marina 1998; Franco y Borrero 2000).

Este escenario se acompaña por una baja intensidad de uso de los pasos naturales del cordón Baguales (Borrero y Carballo Marina 1998; Franco et al. 1999; San Román y Morello 1999) y en otros sectores aledaños como el sistema lacustre al sur del lago Argentino (Franco et al. 1999; también Franco et al. 2011 para el período de ocupación efectiva del área), el Parque Nacional Torres del Paine (Bate 1970; Ortiz Tronoso 1972), y otros lugares hacia el este como las cuencas de los ríos Vizcachas y Zanja Honda (Franco y Borrero 2000), la estancia La Verdadera Argentina (Borrero et al. 2006) y la cuenca media del río Coyle (Belardi et al. 2006). Tanto las mesetas como los cerros aledaños al cordón Baguales pudieron tener un carácter de uso estacional (Borrero et al. 2006; Morano Büchner et al. 2009). Entre los espacios de la periferia meridional $-\mathrm{y}$ que mantuvieron contacto con la sierra Baguales- la obsidiana gris verdosaveteada se registra tempranamente en los sitios Fell y Pali Aike del CVPA, en contextos atribuidos al período cultural III, datado por Bird (1988) entre 8.500 y 6.500 a.p. (Stern y Franco 2000). También fue hallada en los depósitos más antiguos del sitio Cerro Castillo 1 de Última Esperanza, desde el 4.600 a.p. (Langlais y Morello 2009; Legoupil 2009; San Román y Morello 2003), y más tardíamente como parte del ajuar funerario del chenque Cerro Guido (Morano Büchner et al. 2009).

Desde el punto de vista de la circulación, el panorama arqueológico general para el área ha sido parte de la discusión sobre las características biogeográficas del sistema lacustre al sur de Lago Argentino, debido a que este sector configura un callejón sin salida (Borrero 2004), motivado por la presencia del hielo continental y del cordón Baguales (Borrero et al. 2006). Ya para tiempos históricos (fines del siglo XIX y principios del siglo $\mathrm{XX}$ ), los relatos de viajeros destacan el uso de los valles fluviales del río Vizcachas, el curso superior del río Coyle y la naciente del río Gallegos como vías de circulación (ver Borrero y Borrazzo 2011; Martinic 1995, 2004; Musters 2005 [1869-1970]). Algunos de ellos advierten sobre la facilidad del cruce de los ríos durante el invierno, cuando sus aguas se congelaban (Peláez y Jiménez 2006). También la espesura del bosque y los pantanos tenían un importante significado, impidiendo el traslado por lugares como el valle del río de las Chinas o el cruce de las Llanuras de Diana (Martinic 1995, 2004; Musters 2005 [1869-1970]). Otro tanto ha sido mencionado para la cubierta de nieve durante el invierno que hacía intransitables las mesetas al sur de la sierra Baguales (Borrero y Borrazzo 2011). Estos espacios también participaron de los circuitos de movilidad e interacción de los aonikenk, asociados con una ruta tradicional que unía la sierra Baguales con la costa oeste del estrecho de Magallanes y con un asentamiento indígena sobre el valle medio del río Vizcachas (Martinic 1995; Massone 1984).

Las interacciones humanas con la sierra Baguales funcionaron dentro de una amplia red con formas de explotación logística a partir de rangos de acción centrados en ambientes de estepa (Borrero et al. 2006; Charlin et al. 2011). Entre ellos, se destaca el CVPA por su alta intensidad ocupacional y por la persistencia en la explotación de una fuente de obsidiana distante $(>200 \mathrm{~km})$ y de acceso probablemente estacional. Esto le confiere a las vías de acceso a la sierra Baguales un fuerte significado biogeográfico para comprender los alcances y limitaciones de la circulación humana a grandes distancias, vinculadas con importantes procesos de cambio ambiental durante el Holoceno.

\section{Metodología}

La circulación conlleva un gasto de energía que varía en función de lo que se está transportando, de la distancia que se recorre y el terreno que se cubre (Kelly 1992). Así, las posibilidades de la circulación humana necesitan ser evaluadas a partir de simular la estructura del paisaje. En este caso, con un entorno SIG (software ESRI ArcMap 9.3) se combinaron capas de información sobre altura y pendiente del terreno (derivadas del DEM del 
proyecto Shutter Radar Topography Mission-SRTMde la NASA), comunidades vegetales (Luerbert y Pliscoff 2009; Oliva et al. 2001), fuentes y cursos de agua permanentes (proyecto Atlas de la República Argentina del Instituto Geográfico Nacional 2011 y del Sistema Nacional de Información Ambiental de Chile 2010) y presencia de nieves eternas en este sector de la cordillera de los Andes durante el verano (Rivera 2005). Cada uno de estos factores presenta grados de permeabilidad y transitabilidad por lo que no debieran ser considerados barreras geográficas (p.ej., Borrero y Borrazzo 2011; Boschin y Nacuzzi 1979; Musters 2005 [1869-1970]), aunque se espera que en ciertas circunstancias hayan sido evitados como vías regularmente usadas.

Para el área de estudio, las zonas de muy alto costo han sido consideradas aquellas con pendientes mayores a $40^{\circ}$, alturas superiores a los $1.400 \mathrm{msm}$ -que representan el límite aproximado del hielo y las nieves eternas durante el verano, sensu Rivera 2005-, la vegetación de bosque siempreverde, y los cuerpos de agua permanentes que cubren un área superior a medio kilómetro. Los restantes espacios tienen costos menores, que fueron sopesados de manera ordinal en función de las variaciones que presentan cada una de las capas temáticas.

Entre la sierra Baguales, tomando un punto medio aproximado para el área de disponibilidad de la obsidiana gris verdosa veteada (Stern y Franco 2000) y los sitios del CVPA, se consideraron las rutas de menor costo por medio del módulo Shortest Past del Spatial Analysis. Las rutas se calcularon sobre una superficie de fricción basada en un modelo isotrópico, en el cual la fricción es independiente de la dirección del movimiento. Sobre esta base se testearon distintas alternativas de superficies de fricción que permitieron evaluar comparativamente la importancia de cada una de las variables ambientales, incluyendo las posibilidades de la circulación humana durante el verano, dado que el modelado también contempla la presencia de nieves eternas para este período (Rivera 2005) y la disponibilidad de fuentes de agua permanentes. En el caso del invierno, se necesitó modelar las condiciones estacionales a partir de capas sobre temperatura media, precipitación (proyecto WorldClim, Hijmans et al. 2005) y velocidad del viento ${ }^{2}$ (Camilloni 2008; Capel Molina 1983) para el trimestre más frío. También se incluyeron valores de presencia de nieve fresca para mediciones realizadas en lugares donde es frecuente encontrar cubierta de nieve y con temperaturas promedio de invierno semejantes (Ventskevich 1961). Estas capas temáticas fueron reclasificadas en cinco rangos de variación y sumadas en un nuevo modelo (ver metodología en Pallo 2012). Finalmente, las rutas de menor costo se superpusieron al modelo invernal a fin de discutir su disponibilidad estacional.

\section{Resultados y Discusión}

A partir de tres modelos diferentes de superficies de fricción se obtuvieron múltiples rutas de menor costo que unen sitios del CVPA con la sierra Baguales. Dado que los estudios paleoambientales dan cuenta de la ausencia o alternancia de períodos de bosques más abiertos y más cerrados durante el Holoceno, en el primer modelo se exceptuó la vegetación a fin de evaluar la circulación humana en períodos en que el bosque no funcionó como un factor inhibidor. En el segundo modelo se incluyó la cubierta vegetal, pero se descartó la elevación, mientras que en el tercer modelo se presentan todas las capas temáticas.

El primer modelo reconoce una sola vía de circulación de menor costo posible (Figura 3). En la conexión con Baguales, los sitios del CVPA podrían haber estado comunicados entre sí y con los de la región de Última Esperanza por medio de esta única ruta que incluye un recorrido por la cuenca del río Gallegos, el cruce de las Llanuras de Diana y la subida a Baguales bordeando las estribaciones occidentales del sector continental.

En vistas de la factibilidad de esta ruta, la información paleoambiental permite deducir buenas posibilidades durante la transición PleistocenoHoloceno, debido a las condiciones relativamente secas, previas al comienzo de la expansión del bosque abierto del Holoceno temprano (Tonello et al. 2009; Villa-Martínez y Moreno 2007). Para estos momentos, la cuenca del río Gallegos y las Llanuras de Diana debieron constituir una ruta de bajo costo en la conexión con los espacios transcordilleranos (Borrero y Manzi 2007). Sin embargo, el tramo que bordea el lago El Toro y alcanza la cuenca del río de Las Chinas debió estar inundado por el Gran Paleolago Tehuelche (Solari et al. 2012). Una conexión más clara con Baguales debió establecerse una vez desarrollado el actual complejo hidrológico del área, luego del 7.113 cal a.p. (Solari et al. 2012) y con anterioridad al sostenido crecimiento del bosque de Nothofagus 


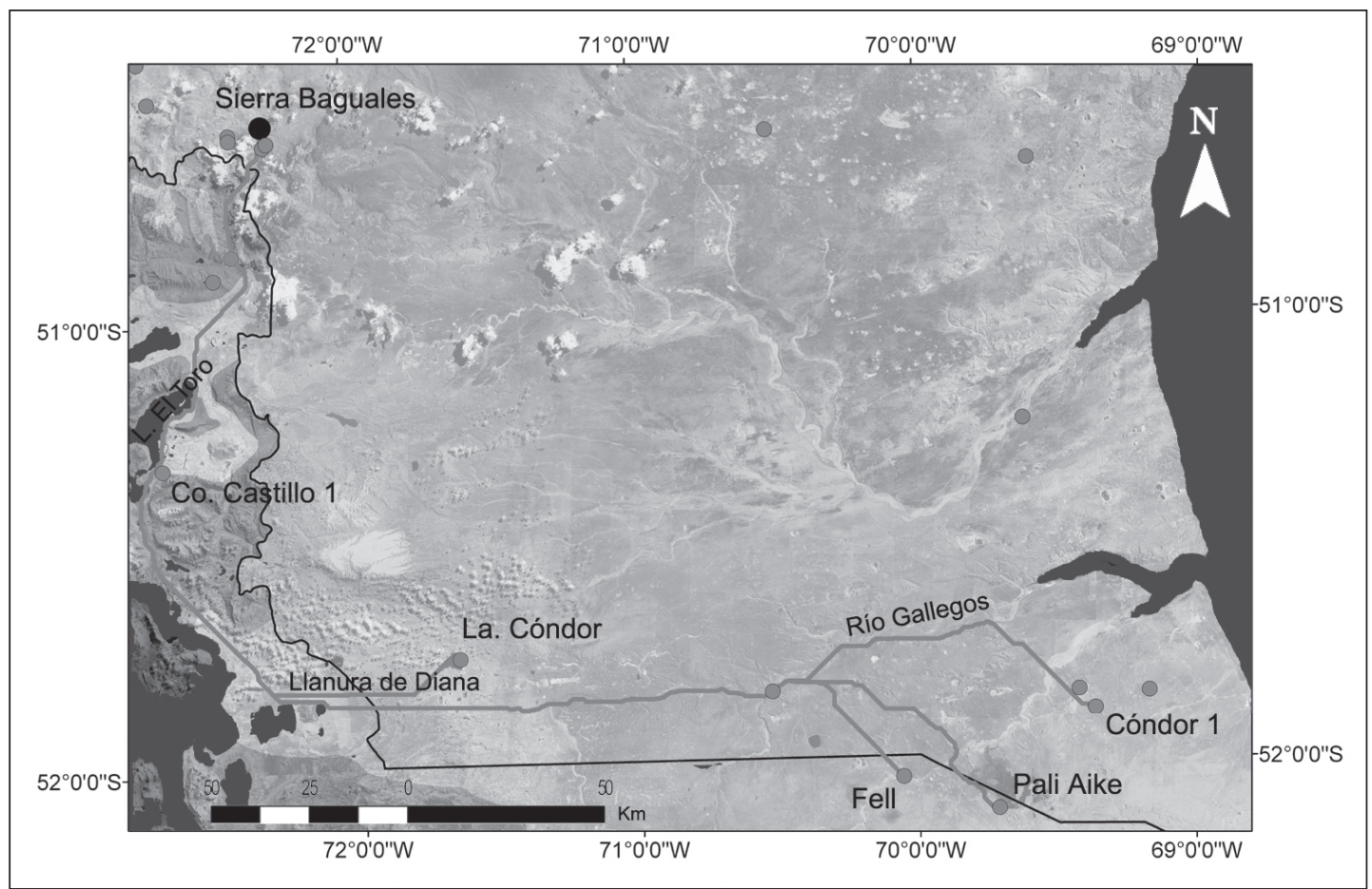

Figura 3. Vías de paso de menor costo generadas con el primer modelo, incluyendo la localización de la potencial fuente Baguales (circulo negro) y los sitios con artefactos manufacturados en obsidiana gris-verdosa veteada (círculos grises).

Least cost routes generated with the first model, including the location of the potential Baguales source (black circle) and the sites with artifacts made of banded greenish-gray obsidian (gray circles).

que comenzó en el 6.800 cal a.p. (Villa-Martínez y Moreno 2007).

De este modo, la primera ruta modelada pudo estar completamente disponible durante el Holoceno Medio, pero en un lapso muy breve. Para ese entonces, la obsidiana gris verdosa ya estaba presente en el CVPA (Stern y Franco 2000), pero las fechas más tempranas de su aparición en el sitio Cerro Castillo 1 son posteriores a la expansión del bosque (San Román y Morello 2003). Estos datos junto con las diferencias tecnológicas observadas entre ambos espacios (Charlin et al. 2011; Langlais y Morello 2009) no alientan a sostener la hipótesis de una ruta occidental usualmente transitada por humanos en la conexión CVPA-sierra Baguales.

A diferencia del primero, el segundo modelo da cuenta de varias vías posibles sin que los sitios del CVPA se comuniquen entre sí en la circulación hacia Baguales (Figura 4). Las rutas traspasan la estepa tendiendo a ser rectas debido a la existencia de una planicie glacifluvial de relieve suave y homogéneo (Oliva et al. 2001). Esto también está asociado con las posibilidades que ofrece la estepa patagónica para circular en múltiples direcciones, en contraposición a lo esperable en ambientes boscosos (Pallo y Borrero 2014).

No obstante esto, los ambientes de estepa semidesértica entre los ríos Gallegos y Coyle se caracterizan por poseer pocos cursos de agua permanente y amplios sectores con pequeñas lagunas y bajos sin salida, muy variables en el corto plazo (Mazzoni et al. 2006). Dicha escasez de agua pudo ser un motivo suficiente para redireccionar la rectitud de los recorridos por estos espacios intermedios. En particular durante períodos secos, una ruta alternativa debió implicar el cruce del río Gallegos hasta alcanzar el tramo más próximo del río Coyle, continuando el traslado por su valle principal. Esta ruta involucra una mayor distancia a recorrer frente a vías de paso más directas hacia la sierra Baguales, pero en el largo plazo debió asegurar la cercanía a una fuente de agua altamente confiable y con buena productividad primaria a su alrededor (Mazzoni y Vázquez 2004).

En relación con esto, las distribuciones arqueológicas en distintas partes del valle del río 


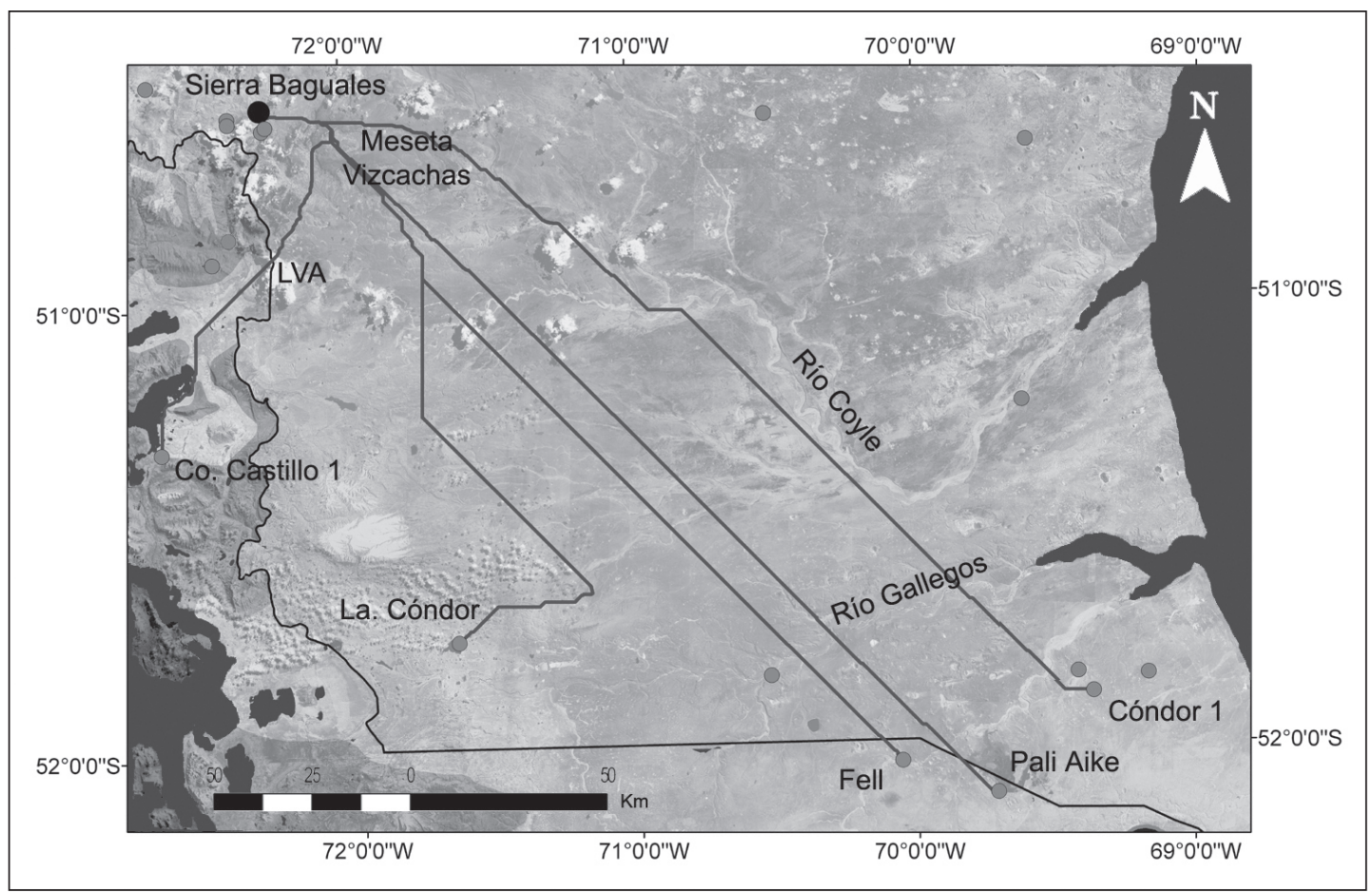

Figura 4. Vías de paso de menor costo elaboradas con el segundo modelo, la fuente de obsidiana Baguales (círculo negro) y la distribución de sitios con presencia de dicha roca (círculos grises).

Least cost routes generated with the second model, the potential Baguales obsidian source (black circle) and the distribution of sites which include such rock (gray circles).

Coyle apuntan a una baja intensidad de ocupación humana que habría implicado el uso preferencial de estos espacios como una importante vía para la circulación (Belardi et al. 2006; Carballo Marina et al. 2011; ver también Martinic 2004 para tiempos históricos). Incluso, los períodos de congelamiento del río (para tiempos históricos ver Peláez y Jiménez 2006) pudieron ofrecer buenas posibilidades para el traslado (Borrero 2012a). Por encima del río Coyle, las diferentes vías de paso confluyen en privilegiar un acceso a la sierra Baguales por las estribaciones orientales, a través de la meseta Vizcachas. Respecto de estos espacios, es sabido que -al menos a fines del siglo XIX-participaron de los circuitos de movilidad de los aonikenk y de las rutas de viaje de otros exploradores hacia el sur del lago Argentino (Martinic 2004). En términos paleoambientales, estos espacios también pudieron estar disponibles con anterioridad.

El modelo también admite al área de LVA como un posible paso transcordillerano de bajo costo en la conexión entre Baguales y los sectores occidentales (sitio Cerro Castillo 1). Sin embargo, hemos señalado que la tendencia distribucional de la obsidiana gris-verdosa veteada para el área desalienta esta alternativa, sumado a que el panorama más general de la información arqueológica sugiere que el cordón Baguales no funcionó como una zona usual de paso hacia la vertiente pacífica (Borrero y Franco 2000).

El tercer modelo se asemeja al segundo en cuanto a la elección de rutas rectilíneas por ambientes de estepa, aunque en ciertos casos se privilegió el sorteo de elevaciones amesetadas por sectores más bajos (Figura 5). La entrada al sector montañoso marca la mayor diferencia con el modelo anterior, debido a que en este último caso se generaron vías de paso que bordean el río Vizcachas por el sur o la meseta homónima, siguiendo la cuenca del río Pelque por el norte. Aquí la inclusión del factor elevación favoreció una mayor cercanía de las vías de paso a los valles fluviales de los ríos Coyle, Vizcachas y Pelque. Al menos en ciertos períodos -asociados con la necesidad humana de disponer de agua- las vías del tercer modelo pudieron tener una mayor aplicabilidad real que las modeladas anteriormente. 


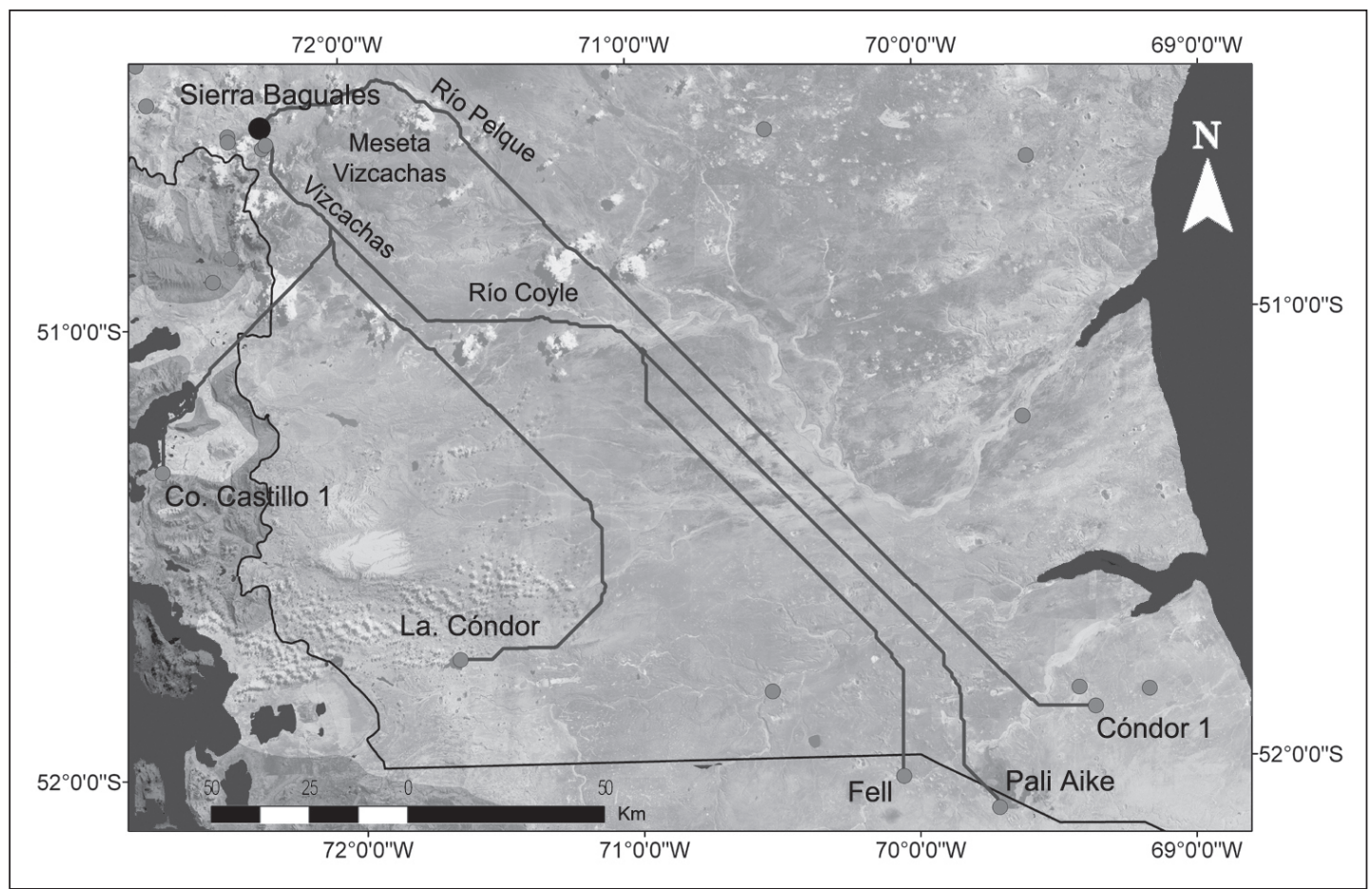

Figura 5. Vías de paso derivadas del tercer modelo, la potencial fuente Baguales (círculo negro) y los sitios con obsidiana grisverdosa veteada (círculos grises).

Least cost routes derived from the third model, the potential Baguales source (black circle) and the sites with banded greenishgray obsidian ( gray circles).

Al contrastar los distintos modelos con la simulación de estrés invernal, se observa que la mayor parte de los recorridos se asocian con niveles relativamente bajos (Figura 6a). Una vez alcanzadas las estribaciones de la sierra Baguales, la primera vía modelada incluye el tramo comparativamente más corto vinculado con niveles de alto estrés invernal (> valor 15), pero transitando zonas más extremas respecto de los otros modelos (Figura 6b). Las vías del segundo modelo dan cuenta de los tramos más largos con alto estrés invernal por la meseta Vizcachas, y las del tercer modelo sostienen niveles de estrés similares al del segundo, aunque en tramos más cortos dado que traspasan sectores más bajos y próximos a los valles fluviales que bordean la meseta Vizcachas.

De acuerdo con esto, la mitad oeste de la sierra Baguales sería el sector más afectado por el aumento de las condiciones invernales, implicando costos máximos de acceso a la fuente. También, es probable que durante el invierno la meseta constituyera una barrera relativamente más importante que otros pasos naturales hacia la sierra Baguales, en particular por las grandes distancias a recorrer asociadas a niveles altos de estrés. Si existieron vías naturales que pudieron ser transitadas todo el año para entrar a la fuente de obsidiana (Borrero y Borrazzo 2011), el modelado invernal sostiene una mayor correspondencia con los valles fluviales que corren por el norte del área, como el arroyo de la Cumbre o del Medio y el río Centinela. Por su parte, la información arqueológica señala que estos cursos de agua alcanzan las localidades arqueológicas Altas Cumbres y Cerro Verlika -respectivamente-, muy próximas al área de aprovisionamiento estimada para la obsidiana gris-verdosa veteada (Franco et al.1999; Stern y Franco 2000). De manera hipotética, las rutas que entraban por las estribaciones orientales pudieron hacer frente a las dificultades impuestas por el aumento del estrés invernal, desviándose hacia el norte mediante la circunvalación de los sectores bajos del cordón Baguales. Aunque un redireccionamiento de este tipo debió elevar notablemente las distancias del recorrido y por ende los costos del traslado, esta pudo ser una opción relativamente poco costosa en períodos muy fríos. 


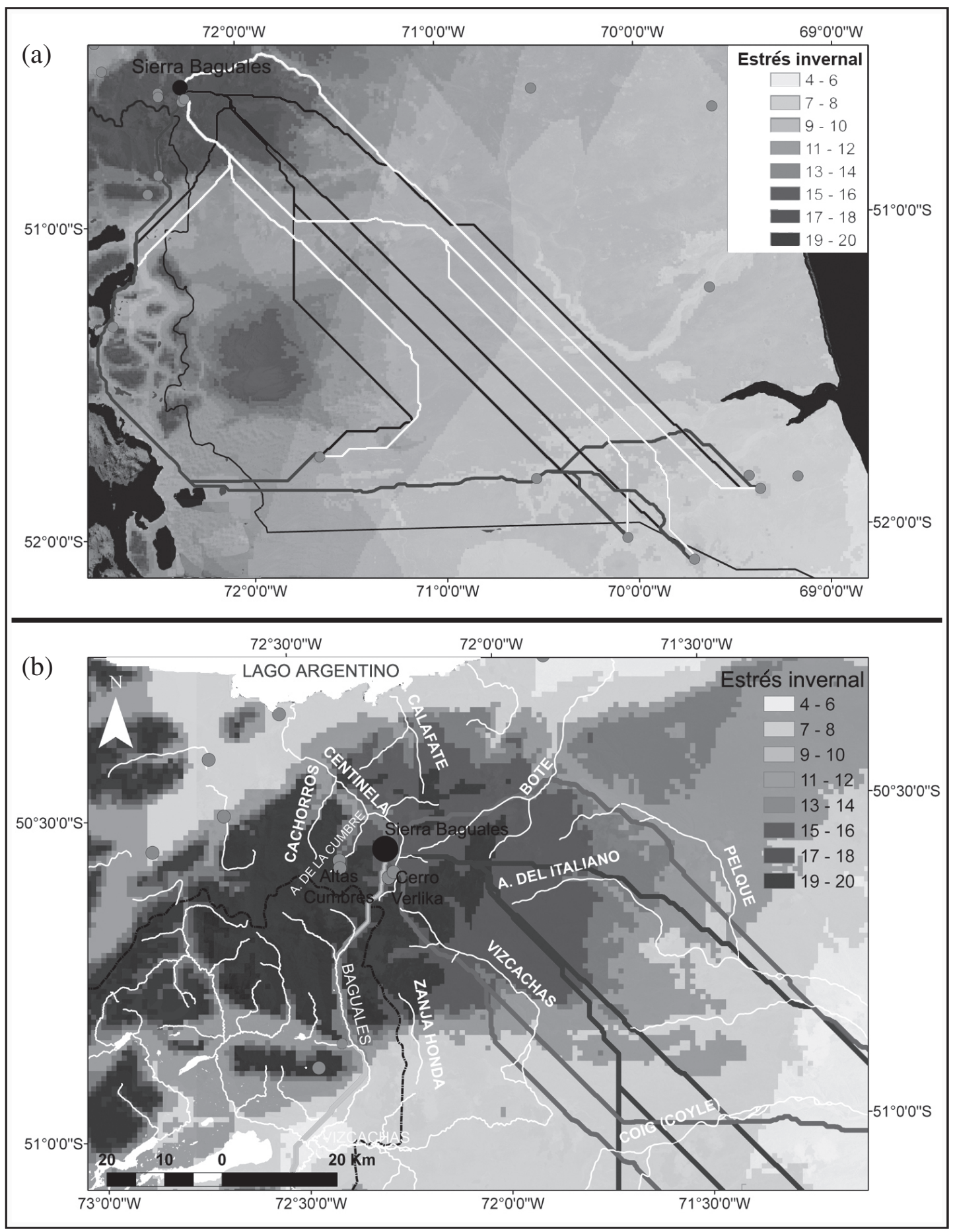

Figura 6. (a) Vías de paso de menor costo (modelos uno, dos y tres) en relación con el estrés invernal (arriba) y (b) visión en escala amplia del acceso a la sierra Baguales.

(a) Least cost routes (models one, two and three) and (b) vision on wide-scale from the access to the Baguales range. 


\section{Conclusión}

El desarrollo de distintas superficies de fricción demostró ser una metodología útil para evaluar la circulación humana entre el CVPA y la sierra Baguales, sopesando potenciales escenarios de rutas y ambientes cambiantes durante el Holoceno. En función de esto es posible plantear que entre la retirada del paisaje glaciar y la expansión boscosa, los espacios occidentales debieron implicar bajo costo de traslado en dirección norte-sur; aunque su disponibilidad pudo ser muy breve. Con posterioridad, el bosque debió ser determinante en el acceso hacia el oeste y el norte del área, reforzando el uso de vías alternativas de bajo costo por las estepas del este.

En momentos húmedos un trazado de rutas esteparias -privilegiando la rectitud y el acortar distancias durante el recorrido-sería suficiente para explicar la conexión entre el CVPA y Baguales. Durante períodos secos surge la hipótesis del uso regular de zonas bajas asociadas con fuentes de agua confiable, y en particular del valle del río Coyle como principal vía de conexión con Baguales. También, al aumentar el estrés invernal debieron amplificarse los costos de la circulación humana, más aún en zonas montañosas. Vías de entrada alternativas con una mayor disponibilidad anual a la fuente de obsidiana pudieron ser puestas en uso (p.ej., valles fluviales del norte), mientras que zonas de alto estrés invernal debieron ser descartadas (p.ej., las mesetas al sureste de Baguales).

Estos resultados apuntan a que la circulación humana hacia Baguales no puede ser explicada por una única vía de bajo costo; también desestiman la importancia de una posible conexión entre el CVPA y Baguales a través del borde occidental, y priorizan una entrada por las estepas orientales. Un pool de rutas de bajo costo -cuya optimalidad fue variable tanto en la escala corta como larga de los cambios paleoambientales del Holocenoparece la hipótesis más defendible. Además, es claro que resulta difícil generar un acercamiento profundo a los patrones temporales, aunque las alternativas aquí planteadas son parte del conocimiento para avanzar en el estudio de esta clase de fuentes de obsidiana-de acceso difícil y estacional-, que permiten especular que se trata de zonas que fueron elegidas por otras razones y que una vez descubierta la obsidiana se crearon condiciones de planeamiento -especial o embedded (sensu Binford 1979) - para obtenerla. Es decir, la obsidiana pudo pesar en el retorno a esos lugares, generando condiciones de fidelidad territorial (Borrero 2012b:287).

Aunque colateralmente, también han quedado esbozados posibles vectores de acceso independiente a la sierra Baguales, desde el sureste (Laguna Cóndor-CVPA) y desde Última Esperanza. Frente a los circuitos por zonas de estepa extra-andina del primer caso, para el segundo queda planteada una hipotética ruta occidental a través de los valles de los ríos De las Chinas y Baguales, derivada del primer modelo. La información arqueológica recopilada hasta el momento es alentadora en este sentido (Borrero et al. 2006; Franco y Borrero 2000; San Román y Morello 1999). Esta ruta occidental debió implicar altos costos de traslado (vinculada con densos bosques, pantanos, desbordes de ríos o caída de nieve), lo que sumado a la menor estacionalidad del corredor oriental, servirían para zanjar la cuestión acerca de la intensidad diferencial en el uso de la obsidiana de Baguales entre los sitios del borde occidental y el CVPA. En la escala regional más amplia, se sigue reforzando la existencia de una circulación asimétrica en la que Última Esperanza habría sido utilizada de manera marginal durante el Holoceno Tardío, tanto desde las tierras del este como desde el sector de canales (Borrero et al. 2006).

Agradecimientos: A Luis Borrero por su constante apoyo, atentas lecturas y comentarios. A los evaluadores de este artículo, quienes aportaron valiosas sugerencias para mejorarlo. Este trabajo es parte de una beca postdoctoral otorgada y financiada por CONICET (Argentina). 


\section{Referencias Citadas}

Avery, D. 1995. Physical environment and site choice in South Africa. Journal of Archaeological Science 22:343-353.

Bate, L. 1970. Primeras investigaciones sobre el arte rupestre de la Patagonia. Anales del Instituto de la Patagonia 1:15-26.

Beaton, J. 1991. Colonizing continents: some problems from Australia and the Americas. En The First Americans: Search and Research, editado por T. Dillehay y D.J. Meltzer, pp. 209-230. CRC Press, Boca Raton.

Belardi, J., F. Carballo Marina y S. Espinosa (eds.) 2006. Cazadores recolectores en la cuenca media e inferior del río Coyle. En $L a$ Cuenca del Río Coyle. Estado Actual de las Investigaciones, pp. 97-21-28. UNPA, Río Gallegos.

Binford, L. 1979. Organization and formation processes: looking at curated technologies. Journal of Anthropological Research 35:255-273.

Binford, L. 1982. The archaeology of place. Journal of Anthropological Archaeology 1:5-31.

Bird, J. 1988. Travels and Archaeology in South Chile, editado por J. Hyslop. University of Iowa Press, Iowa.

Borrazzo, K. 2006. Tecnología lítica del alero Cerro León 3. Magallania 34:63-74.

Borrazzo, K. 2008. Análisis tecnológico de distribuciones artefactuales en la periferia sudeste de la Sierra Baguales. Magallania 36:103-116.

Borrero, L. 1989-90. Evolución cultural divergente en la Patagonia austral. Anales del Instituto de la Patagonia 19:133-139.

Borrero, L. 2004. The Archaeozoology of andean 'dead ends' in Patagonia: living near the continental ice cap. En Colonisation, Migration and Marginal Areas. A Zooarchaeological Approach, editado por M. Mondini, S. Muñoz y S. Wickler, pp. 55-61. Oxbow Books, Oakville.

Borrero, L. 2012a. The human colonization of the high Andes and southern South America during the cold pulses of the late Pleistocene. En Hunter-Gatherer Behavior: Human Response during the Younger Dryas, editado por M. Eren, pp. 57-78. Left Coast Press, California.

Borrero, L. 2012b. La Patagonia cuyana. En Logros y Desafíos. Arqueología y Paleoecología de Cuyo, editado por A. Gil y G. Neme, pp. 282-295. SAA, Buenos Aires.

Borrero, L. y K. Borrazzo (eds.) 2011. La geografía cultural del sudoeste de Patagonia Continental. En Bosques, Montañas y Cazadores: Investigaciones Arqueológicas en Patagonia Meridional, pp. 7-36. Dunken, Buenos Aires.

Borrero, L. y F. Carballo Marina 1998. Proyecto Magallania: la cuenca superior del río Santa Cruz. En Arqueología de la Patagonia Meridional. Proyecto Magallania, editado por L. Borrero, pp. 11-27. Búsqueda de Ayllu, Concepción del Uruguay.

Borrero, L. y N. Franco 2000. Cuenca superior del río Santa Cruz: perspectivas temporales. En Desde el País de los Gigantes. Perspectivas Arqueológicas en Patagonia, editado por J. Belardi, F. Carballo Marina y S. Espinosa, pp. 345-356. UNPA. Río Gallegos.
Borrero, L., N. Franco, F. Martin, R. Barberena, R. Guichon, J. Belardi, C. Favier Dubois y L. L'Heureux 2006. Las Cabeceras del Coyle: información arqueológica y circulación de poblaciones humanas. En Pasado y Presente en la Cuenca del Río Coyle, editado por F. Carballo Marina, J. Belardi y S. Espinosa, pp. 75-95. UNPA, Río Gallegos.

Borrero, L. y L. Manzi 2007. Arqueología supraregional y biogeografía en Patagonia meridional. En Arqueología de Fuego-Patagonia. Levantando Piedras, Desenterrando Huesos... y Develando Arcanos, editado por F. Morello, M. Martinic, A. Prieto y G. Bahamonde, pp. 411-419. Ediciones CEQUA, Punta Arenas.

Boschín, M. y L. Nacuzzi 1979. Ensayo metodológico para la reconstrucción etnohistórica. Su aplicación a la comprensión del modelo tehuelche meridional. Colegio de Graduados en Antropología. Serie Monográfica $N^{\circ} 4$, Buenos Aires.

Camilloni, I. 2008. Atlas de Sensibilidad Ambiental de la Costa y el Mar Argentino. UNDP. http://atlas.ambiente.gov.ar (2 de abril de 2008)

Capel Molina, J. 1983. Reflexiones geográficas acerca del clima frío oceánico del hemisferio sur, Punta Arenas. Revista de Geografía Norte Grande 10:3-16.

Carballo Marina, F., J. Belardi y J. Sáez 2011. Distribución espacial del registro arqueológico en la unidad de paisaje terrazas, cuenca media del río Coyle. Magallania 39:209-224.

Charlin, J. 2009. Estrategias de Aprovisionamiento y Utilización de las Materias Primas Líticas en el Campo Volcánico Pali Aike. Archaeopress (BAR 1901), Oxford.

Charlin, J., L. Borrero y C. Pallo 2011. Ocupaciones humanas en el área noroccidental del río Gallegos. En Bosques, Montañas y Cazadores: Investigaciones Arqueológicas en Patagonia Meridional, editado por L. Borrero y K. Borrazo, pp. 179-210. Dunken, Buenos Aires.

Franco, N. 2014. Lithic artifacts and the information about human utilization of large areas. En Artefactos Líticos, Movilidad y Funcionalidad de Sitios: Problemas y Perspectivas, editado por P. Escola y S. Hocsman, pp. 117-127. British Archaeological Reports, Oxford.

Franco N. y L. Borrero 2000. Estrategias de utilización de Sierra Baguales. Actas XIV Congreso Nacional de Arqueología Chilena. Contribución Arqueológica 5:269-283.

Franco N. y L. Borrero 2003. Chorrillo Malo 2: initial peopling of the Upper Santa Cruz Basin, Argentina. En Where the South Winds Blow. Ancient Evidence of Paleo South Americans, editado por L. Miotti, M. Salemme y N. Flegenheimer, pp. 149- 152. Center for the study of the first Americans y Texas A \& M University Press, Texas.

Franco, N., L. Borrero, J. Belardi, F. Carballo Marina, F. Martín, P. Campán, C. Favier Dubois, N. Stadler, M. Hernandez Llosas, H. Cepeda, S. Muñoz, F. Borella, e I. Cruz 1999. Arqueología del Cordón Baguales y Sistema Lacustre al Sur del Lago Argentino. Praehistoria 3:65-86.

Franco, N., N. Cirigliano y P. Ambrústolo 2011. Semejanzas en tecnologías, diseños y prácticas funerarias al sur de la cuenca 
superior del río Santa Cruz: algunos ejemplos correspondientes al Holoceno tardío. En Bosques, Montañas y Cazadores: Investigaciones Arqueológicas en Patagonia Meridional, editado por L. Borrero y K. Borrazo, pp. 155-178. Dunken, Buenos Aires.

Gamble, C. 1998. Concluding remarks: archaeology's fifth big question. En Early Human Behaviour in Global Context. The Rise and Diversity of the Lower Paleolithic Record, editado por M. Petraglia y R. Korisettar, pp. 451-467. One World Archaeology Series 28. Taylor \& Francis, Londres.

García, J., B. Hall, M. Kaplan, R. Vega, y J. Strelin 2014. Glacial geomorphology of the Torres del Paine region: implications for glaciation, deglaciation and paleolake history. Geomorphology 204:599-616.

Glasser, N., S. Harrison, V. Winchester y M. Aniya 2004. Late Pleistocene and Holocene glacier fluctuations in Patagonia. Global and Planetary Change 43:79-101.

Hijmans, R., S. Cameron, J. Parra, P. Jones y A. Jarvis 2005. Very high resolution interpolated climate surfaces for global land areas. International Journal of Climatology 25:1965-1978.

Huber, U. y V. Markgraf 2003. European impact on fire regimes and vegetation dynamics at the steppe-forest ecotone of southern Patagonia. The Holocene 13:567-579.

Huber, U., V. Markgraf y F. Schäbitz 2004. Geographical and temporal trends in Late Quaternary fire histories of FuegoPatagonia, South America. Quaternary Science Reviews 23:1079-1097.

Ingold, T. 2000. The Perception of the Environment. Routledge, London.

Kelly, R. 1992. Mobility/Sedentism: concepts, archaeological measures and effects. Annual Review in Anthropology 21:43-66.

Kelly, R. 1995. The Foraging Spectrum: Diversity in HunterGatherer Lifeways. Smithsonian Institution Press, Washington DC.

Langlais, M. y F. Morello 2009. Estudio tecno-económico de la industria lítica de Cerro Castillo (Última Esperanza, Chile). Magallania 37:61-84.

Legoupil, D. 2009. La ocupación del alero Cerro Castillo en la antigua cuenca glaciar del Lago Toro hacia $\mathrm{V}^{\circ}$ millenio AP. Magallania 37:19-38.

Luerbert, F. y P. Pliscoff 2009. Sinopsis bioclimática y vegetacional de Chile. http://territorial.sinia.cl (2 de septiembre de 2009).

Madry, S. y L. Rakos 1996. Line-of-sight and cost surface techniques for regional archaeological research in the Arroux River Valley. En New Methods, Old Problems: Geographic Information Systems in Modern Archaeological Research, editado por H. Maschner, pp. 104-126. CAI Occasional Paper 23. Carbondale, Ilinois.

Mancini, M., F. Bamonte y G. Sottile 2011. Paleoecología y ocupaciones humanas durante el Holoceno en el área cordillerana y Patagonia extra-andina entre los $49^{\circ}$ y $51^{\circ} \mathrm{S}$, Santa Cruz. En Bosques, Montañas y Cazadores: Investigaciones Arqueológicas en Patagonia Meridional, editado por L. Borrero y K. Borrazzo, pp. 37-59. Dunken, Buenos Aires.

Martinic, M. 1995. Los Aónikenk. Historia y Cultura. Universidad de Magallanes, Punta Arenas.
Martinic, M. 2004. Viajeros desconocidos en la Patagonia Austral durante la década de 1870. Magallania 32:5-13.

Massone, M. 1984. Los paraderos Tehuelches y Proto Tehuelches en la costa del Estrecho de Magallanes. Anales del Instituto de la Patagonia 15:27-42.

Matteucci, S., V. Scheinsohn, F. Rizzo y S. Leonardt 2011. Rutas de comunicación trasandina de los cazadores recolectores en el centro-oeste de Chubut. GEOSIG 3:183-200.

Mayr, C., A. Lücke, W. Stichler, P. Trimborn, B. Ercolano, G. Oliva, C. Ohlendorf, J. Soto, M. Fey, T. Haberzettl, S. Janssen, F. Schäbitz, G. Schleser, M. Wille y B. Zolitschka 2007. Precipitation origin and evaporation of lakes in semi-arid Patagonia (Argentina) inferred from stable isotopes $(\delta 18 \mathrm{O}, \delta 2 \mathrm{H})$. Journal of Hydrology 334:53-63.

Mazzoni, E. y M. Vázquez 2004. Ecosistemas de Mallines y Paisajes de la Patagonia Austral. INTA, Río Gallegos.

Mazzoni, E., M. Vázquez, D. Grima y F. Cola 2006. Lake level fluctuations during the last decades in southern Patagonia - an approach using satellite images. Terra Nostra 1:18-19.

Morano Büchner, S., V. Sierpe y A. Prieto 2009. Rescate del "Cheque Cerro Guido". En Arqueología de Patagonia: Una Mirada desde el Último Confín, editado por M. Salemme, F. Santiago, M. Vázquez, E. Piana, M. Álvarez y M. Mansur, pp. 661-668. Utopías, Ushuaia.

Moreno, P., J. Francois, R. Villa-Martínez y C. Moy 2009. Millenial-scale variability in the Southern Hemisphere westerly wind activity over the last 5000 years in SW Patagonia. Quaternary Science Reviews 28:25-38.

Musters, G. 2005 [1869-1970]. Vida entre los Patagones. El Elefante Blanco, Buenos Aires.

Oliva, G., L. González, P. Rial y E. Livrachi 2001. Áreas ecológicas de Santa Cruz y Tierra del Fuego. En Ganadería Ovina Sustentable en la Patagonia Austral. Tecnología de Manejo Extensivo, editado por P. Borrelli y G. Oliva, pp. 41-82. INTA, Buenos Aires.

Ortiz-Troncoso, O. 1972. Material lítico de Patagonia austral. Seis yacimientos de superficie. Anales del Instituto de la Patagonia 3:49-65.

Pafundi, L. 2006. Implicancias Arqueológicas y Modelizaciones Gráficas de los Endicamientos del Sistema Lacustre al Sur de Lago Argentino. Tesis de licenciatura, FFyL-UBA, Buenos Aires.

Pallo, C. 2012. El estrés invernal como generador de áreas marginales en Patagonia meridional durante el Holoceno tardío. Comechingonia Virtual VI:86-114.

Pallo, C. y L. Borrero 2014. Arqueología de corredores boscosos en Patagonia Meridional: el caso del río Guillermo. Intersecciones en Antropología, en prensa.

Paruelo, J., A. Beltrán, E. Jobbágy, O. Sala y R. Golluscio 1998. The climate of Patagonia: general patterns and controls on biotic processes. Ecología Austral 8:85-101.

Peláez, P. y L. Jiménez 2006. Los viajeros en el Coy. En La Cuenca del Río Coyle. Estado Actual de las Investigaciones, 
editado por J. Belardi, F. Carballo Marina y S. Espinosa, pp. 129164. UNPA, Río Gallegos.

Rivera, A. 2005. Cambio climático. Los glaciares de la Patagonia. Investigación y Ciencia 349:36-37.

Ruiz y Doberti Ltda. 2008. Inventario de Atractivos, Recursos, Servicios Turísticos y Recreativos de la Comuna Torres del Payne. Gobierno de Chile, Santiago.

San Román, M. y F. Morello 1999. Caracterización arqueológica preliminar de la cuenca del río Baguales. Anales del Instituto de la Patagonia 27:199-208.

San Román, M. y F. Morello 2003. Nota sobre una excavación de sondeo en el alero Cerro Castillo 1. Magallania 31:139-148.

Sottile, G., F. Bamonte, M. Mancini y M. Bianchi 2012. Insights into Holocene vegetation and climate changes at the Southeastern side of the Andes: Nothofagus Forest and Patagonian Steppe fire records. The Holocene special issue 22:1309-1322.

Solari, M., J. Le Roux, F. Hervé, A. Airo y M. Calderón 2012. Evolution of the Great Tehuelche Paleolake in the Torres del Paine National Park of Chilean Patagonia during the Last Glacial Maximum and Holocene. Andean Geology 39:1-21.
Stern, C. y N. Franco 2000. Obsidiana gris verdosa veteada en la cuenca superior del río Santa Cruz, extremo sur de Patagonia. Anales del Instituto de la Patagonia 28:265-273.

Sturzenbaum, P. y P. Borrelli 2001. Manejo de riesgos climáticos. En Ganadería Ovina Sustentable en la Patagonia Austral. Tecnologías de Manejo Extensivo, editado por P. Borrelli y G. Oliva, pp. 255-270. INTA, Buenos Aires.

Tonello, M., M. Mancini y H. Seppä 2009. Quantitative reconstruction of Holocene precipitation changes in Southern Patagonia. Quaternary Research 72:410-420.

Veth, P. 1993. Islands in the interior: the dynamics of prehistoric adaptations within the arid zone of Australia. International Monographs in Prehistory, Ann Arbor.

Villa-Martínez, R. y P. Moreno 2007. Pollen evidence for variations in the southern margin of the westerly winds in SW Patagonia over the last 12,600 years. Quaternary Research 68:400-409.

Whallon, R. 2006. Social networks and information: Non"utilitarian" mobility among hunter gatherers. Journal of Anthropological Archaeology 25:259-270.

Ventskevich, G. 1961. Agrometeorology. National Science Foundation, Washington DC.

\section{Notas}

1 Aunque aún no ha podido ser confirmado, el reciente hallazgo de dos microdesechos en un nuevo sondeo realizado en el sitio Cerro León 3 abre la posibilidad de contar con una representación marginal de la obsidiana de Baguales en el área de La Verdadera Argentina para el Holoceno Tardío (K. Borrazzo com. pers. 2015).
2 En el caso del área del Lago Argentino, no se pudieron registrar valores en la bibliografía por lo que se optó por considerar un valor aproximado de acuerdo con la información disponible para las restantes áreas. 
